En 2011, les Dr Adrian Sury et Renato Tognina ont repris la présidence et la vice-présidence des délégués cantonaux aux questions des assistantes médicales - je me réjouis de cette collaboration. Je tiens à remercier leurs prédécesseurs, le président Emil Schalch et le vice-président Peter Tschudi, pour leur engagement. Je suis ravi qu'ils continuent à œuvrer pour la formation de base et continue des assistantes médicales: Emil Schalch comme président de I'ORTRA Formation profes- sionnelle des assistantes médicales et vice-président de la Commission pour le développement professionnel et la qualité de la formation des assistantes médicales, Peter Tschudi à titre de président de cette dernière, en plus de sa collaboration au sein de I'ORTRA.

Dr Ernst Gähler, vice-président de la FMH, Responsable du domaine Paramedics

\title{
«Les possibilités de travail des assistantes médicales n’ont jamais été aussi variées»
}

\author{
Depuis janvier Adrian Sury préside l'Assemblée des délégués cantonaux aux questions des assistantes médicales \\ de la FMH. Ce médecin de famille et formateur engagé de Locarno considère l'assistante médicale comme \\ une partenaire qualifié. Pour continuer à développer de manière ciblée cette profession attrayante et à \\ responsabilités, il mise sur la collaboration entre les organisations de formation et de perfectionnement.
}

Interview: Fabienne Hohl

Correspondance:

Dr Adrian Sury

via della Posta 44

casella postale 548

CH-6602 Locarno

adrian.sury@hin.ch

Le nouveau président des délégués cantonaux aux questions des assistantes médicales de la FMH, le Dr Adrian Sury, attache de l'importance à une collaboration harmonieuse entre médecins et assistantes médicales.
Depuis le $1^{e r}$ janvier 2011, vous êtes président des délégués cantonaux aux questions des assistantes médicales de la FMH. Qu'est-ce qui vous a amené à présenter votre candidature?

Adrian Sury: Je suis actif depuis 14 ans dans l'Organisation de formation des assistantes médicales du canton du Tessin et depuis lors aussi délégué cantonal aux questions des assistantes médicales. L'expérience acquise durant ces années me permet d'accomplir mes nouvelles tâches avec circonspection. Par ailleurs, nous «latins» ne pouvons pas sans cesse nous plaindre d'être mis de côté - pour ensuite nous soustraire à nos responsabilités lorsqu'une place se libère. Je suis ravi que mon camarade d'études Renato Tognina, originaire du Val

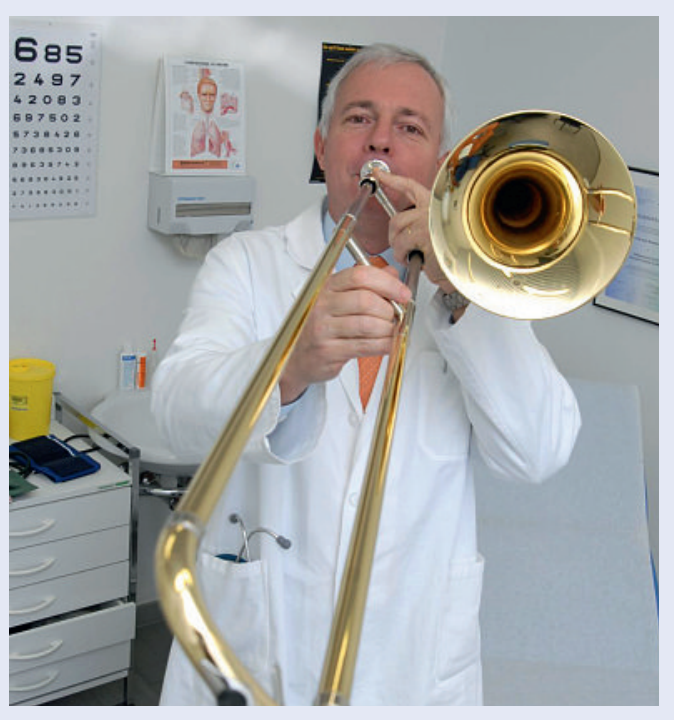

Poschiavo, reprenne la vice-présidence et que la direction soit pour une fois «bilingue» italien-allemand.

Qu'est-ce qui est particulièrement important dans la formation des assistantes médicales?

Je forme des assistantes médicales depuis 1988. J'ai toujours accordé une importance particulière aux capacités communicatives, organisationnelles et humaines de mes assistantes. Elles sont la carte de visite de nos cabinets. Je souhaite leur apprendre à maîtriser les réalités en évolution rapide du domaine de la santé, tout en s'épanouissant au travail.

Où se situe aujourd'hui la profession d'assistante médicale dans le domaine de la santé et en quoi a-t-elle changé?

Les assistantes médicales ne sont plus seulement des aides mais de réelles partenaires, qui apportent des compétences propres au sein de l'équipe du cabinet ou de l'hôpital. Les possibilités de travail et de formation continue n'ont jamais été aussi variées: elles travaillent au cabinet familial, aux urgences ou chez un spécialiste. Dans tous les cas, elles endossent des responsabilités et exercent des tâches très diverses - une profession très variée!

Comment vont évoluer la profession et la formation des assistantes médicales?

Un gros problème concerne la «mortalité professionnelle» des assistantes médicales: beaucoup utilisent cette formation pour accéder à d'autres professions ou fondent une famille. En outre, la discussion sur la pénurie de médecins de famille a engendré de l'insécurité quant à la disponibilité des places de formation 


\section{Personnes et organisations en faveur des assistantes médicales}

En Suisse, plusieurs organisations s'occupent de la profession d'assistante médicale, dont:

- L'Association suisse des assistantes médicales (SVA) s'engage en faveur des assistantes médicales dans le domaine de la formation de base et complémentaire, et les représente sur le plan syndical; présidente centrale: Tresa Stübi - www.sva.ch

- La Fédération Suisse des Associations d'Assistantes Médicales (FSAAM) réunit les organisations professionnelles des assistantes médicales de Genève, de Suisse romande, du Tessin et de Suisse alémanique (Association genevoise des assistantes médicales, Association des assistantes médicales, Asociazione ticinese assistenti di studio medico, Berufsverband medizinischer Praxisassistentinnen); présidente: Elwina Kaufmann - www.bsmpa.ch

- L'Organisation du monde du travail Formation professionnelle des assistantes médicales (ORTRA Formation professionnelle des assistantes médicales) regroupe les associations et les organisations concernées par la formation professionnelle des assistantes médicales et organise des examens professionnels nationaux; président: Emil Schalch - www.odamed.ch

- La Commission suisse pour le développement professionnel et la qualité de la formation des assistantes médicales organise et supervise le plan de formation et l'ordonnance sur la formation; président: Peter Tschudi - www.fmh.ch $\rightarrow$ Services $\rightarrow$ Assistantes médicales $\rightarrow$ Formation

- L'Assemblée des délégués cantonaux aux questions des assistantes médicales de la FMH se consacre aux questions les plus diverses touchant à la profession d'assistante médicale. Les délégués représentent les sociétés cantonales de médecine; président: Adrian Sury - www.fmh.ch $\rightarrow$ Services $\rightarrow$ Assistantes médicales $\rightarrow$ Délégués

et à l'avenir de la profession. En réalité, beaucoup d'assistantes médicales trouvent un poste dans les hôpitaux, qui fournissent de plus en plus de prestations ambulatoires et qui dépendent donc du savoirfaire des assistantes médicales. Nous devons par conséquent inciter les hôpitaux à former eux aussi des assistantes médicales - ce que nous faisons au Tessin depuis plusieurs années déjà, avec beaucoup de succès.

En tant que président, que souhaitez-vous obtenir pour les formatrices et les formateurs?

Une ligne claire et uniforme qui représente les médecins, les assistantes médicales et tous les organes de formation professionnelle dans les questions liées aux assistantes médicales. En effet, des discussions stériles
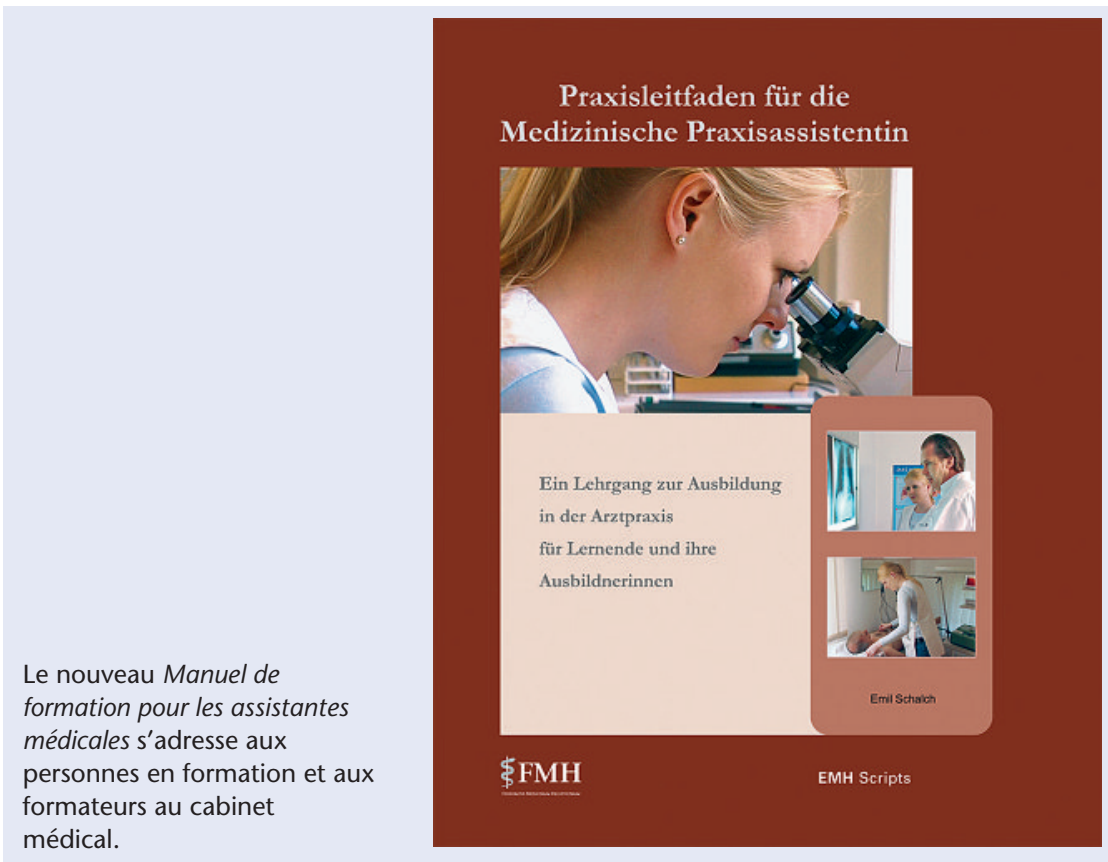

qui, dans l'intérêt des assistantes médicales et des médecins devraient être closes depuis longtemps, subsistent encore. Nous devons impérativement travailler ensemble en vue de consolider cette profession et de l'adapter aux exigences croissantes. Les organisations d'assistantes médicales en particulier doivent en prendre conscience et unir leurs forces pour représenter efficacement un plus grand nombre d'assistantes médicales. Aujourd'hui, beaucoup d'entre elles ne font même pas partie d'une association professionnelle. Il s'agit de les inciter à y adhérer, car pouvoir compter sur des partenaires solides est essentiel dans un contexte politique difficile! Une autre tâche importante sera le financement de la formation continue structurée des assistantes médicales dans le cadre des cours de l'ORTRA.

\section{Commander le manuel de formation et le dossier de formation}

Vous pouvez commander le nouveau Manuel de formation pour les assistantes médicales en allemand au prix de 98 francs (frais d'envoi et TVA en sus) par courriel auprès des éditions médicales suisses: auslieferung@emh.ch. La version française est en cours de traduction.

Vous pouvez obtenir le nouveau Dossier de formation des assistantes médicales en allemand (auparavant journal de travail) auprès du Secrétariat central de l'Association suisse des assistantes médicales (SVA) ainsi que du Secrétariat général de la FMH: info@sva.ch et mpa@fmh.ch, www.fmh.ch $\rightarrow$ Services $\rightarrow$ Assistantes médicales $\rightarrow$ Encadré «Documents à commander». Le classeur coûte 20 francs (frais d'envoi et TVA en sus). 